\title{
Searches for Resonances Decaying to Quarks using the ATLAS Detector
}

\author{
Johannes Erdmann* \\ TU Dortmund University, Germany \\ E-mail: johannes.erdmann@cern.ch \\ on behalf of the ATLAS Collaboration
}

Many theories beyond the Standard Model predict new particles that decay to light or heavy quarks. Such final states are of particular interest at the LHC since new particles produced in parton collisions are likely to produce final states with (at least) two partons. Various strategies are employed to probe high and low resonance masses. The results from latest searches, performed by the ATLAS Collaboration with $13 \mathrm{TeV}$ data, for dijet resonances, for resonances that decay to a top quark and an antitop quark, and for vector-like quarks are presented.

XXVII International Workshop on Deep-Inelastic Scattering and Related Subjects - DIS2019

8-12 April, 2019

Torino, Italy

${ }^{*}$ Speaker. 


\section{Introduction}

The results from a selection of searches for resonances that decay to quarks, performed with the ATLAS detector [1] at the Large Hadron Collider (LHC) in proton-proton collisions at a centerof-mass energy of $13 \mathrm{TeV}$, are presented. The integrated luminosity used in these searches ranges from $36.1 \mathrm{fb}^{-1}$ of data, taken in 2015 and 2016 , to $139 \mathrm{fb}^{-1}$ of data, which correpsonds to the full dataset taken during the LHC Run-2 (2015-2018). The presented results include high-mass dijet searches, dijet searches at lower masses in which events are triggered based on initial-state radiation (ISR) of photons or jets, searches for resonances that decay to a top-quark and an antitop-quark $(t \bar{t})$, and searches for pair- and single-production of vector-like quarks (VLQ).

\section{Dijet resonances}

High-mass dijet resonances were searched for using $139 \mathrm{fb}^{-1}$ of data [2]. Events were triggered with a single-jet trigger with a transverse momentum $\left(p_{\mathrm{T}}\right)$ threshold of $420 \mathrm{GeV}$, and a resonant signal was searched for in the invariant-mass spectrum of the two highest- $p_{\mathrm{T}}$ jets $\left(m_{j j}\right)$ for $m_{j j}>1100 \mathrm{GeV}$. The absolute value of half the rapidity difference between the two jets $\left(\left|y^{*}\right|\right)$ was required to be smaller than 0.6. This suppresses the dominant background, $t$-channel multijet production, which produces more forward jets than the signal process, as the decay of a high-mass resonance often results in two central jets. The background was estimated by fitting $m_{j j}$ with an analytic function. Given the large number of events in the analysed dataset, the so-called "slidingwindow method" was used, fitting only a limited range in $m_{j j}$ around each $m_{j j}$ bin. This method allows for the selection of simpler functions than would be necessary when fitting the full spectrum.
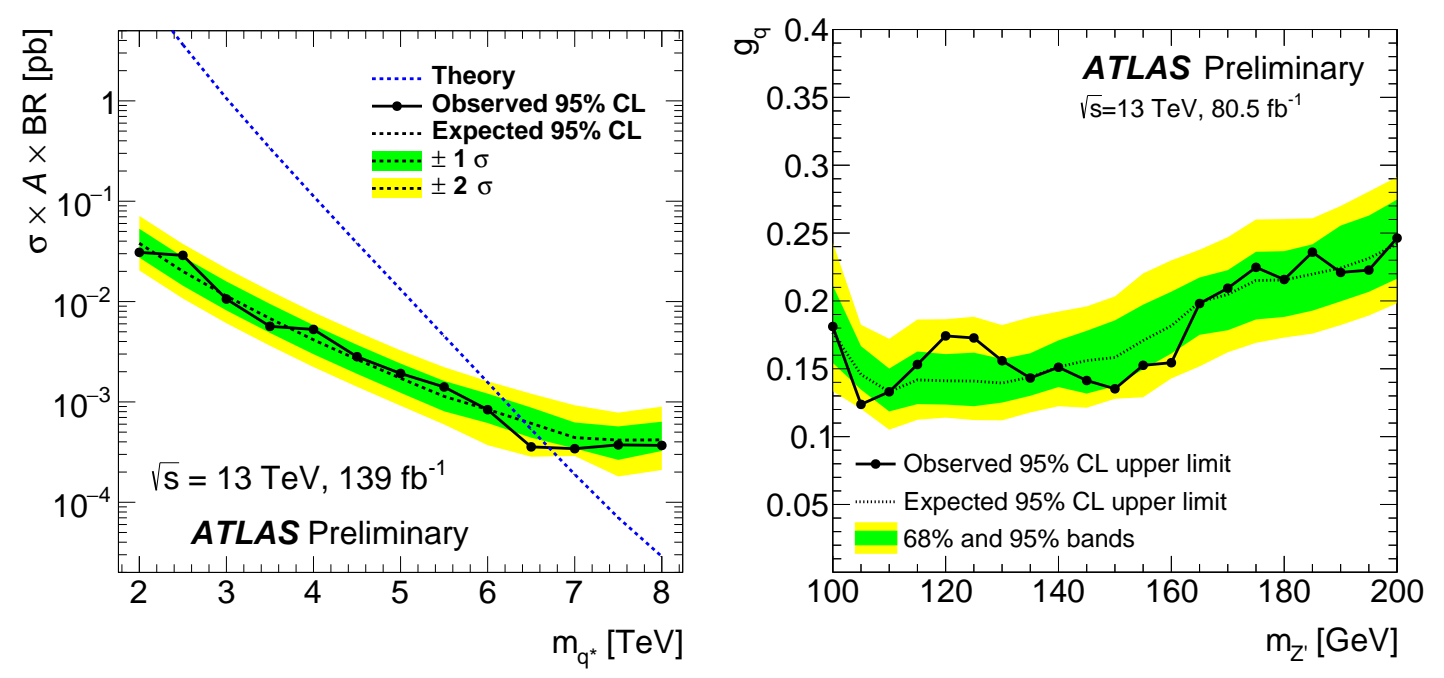

(a)

Figure 1: (a) Upper limit at 95\% CL on the cross section times acceptance and branching ratio for an excited quark, $q^{*}$, as a function of its mass [2]. (b) Upper limit at $95 \%$ credibility level on the coupling of a $Z^{\prime}$ boson to quarks as a function of its mass [5]. 
No significant excess above the Standard Model (SM) background was found and 95\% confidence level (CL) upper limits were set on the cross section times acceptance and branching ratio (BR) for the production of an excited quark, $q^{*}$, as a function of its mass (Figure 1(a)). The corresponding lower mass limit at 95\% CL is $6.7 \mathrm{TeV}$. Limits were also set for a generic signal with a Gaussian shape in $m_{j j}$.

At lower dijet masses, dijet searches are limited by the total rate of the high-level trigger: The minimum- $p_{\mathrm{T}}$ tresholds for single-jet triggers, used to keep the rates for these triggers at an acceptable level, are too high for an efficient search for resonances with masses lower than $1 \mathrm{TeV}$. This issue was addressed by a search for lower-mass dijet resonances by triggering instead on a photon that is produced by ISR in addition to the jets, using up to $79.8 \mathrm{fb}^{-1}$ of data [3]. The single-photon trigger was complemented by a combined trigger that allowed to lower the photon- $p_{\mathrm{T}}$ treshold but required two additional jets. While the combined trigger assures a higher signal acceptance at high resonance masses it loses acceptance at low resonance masses due to the minimum- $p_{\mathrm{T}}$ thresholds on the jets in the trigger.

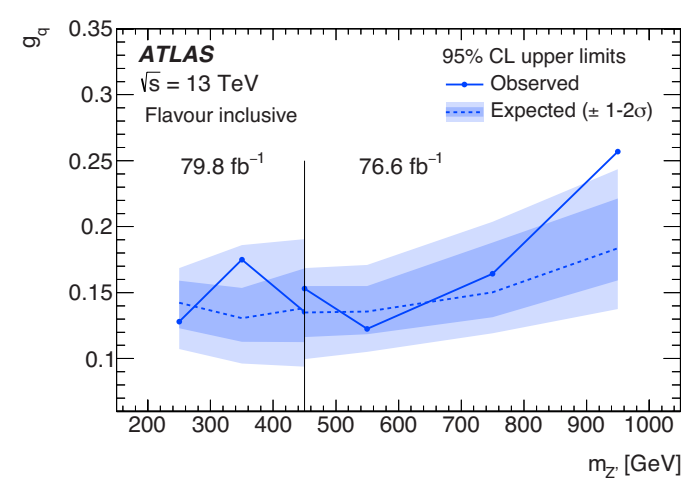

(a)

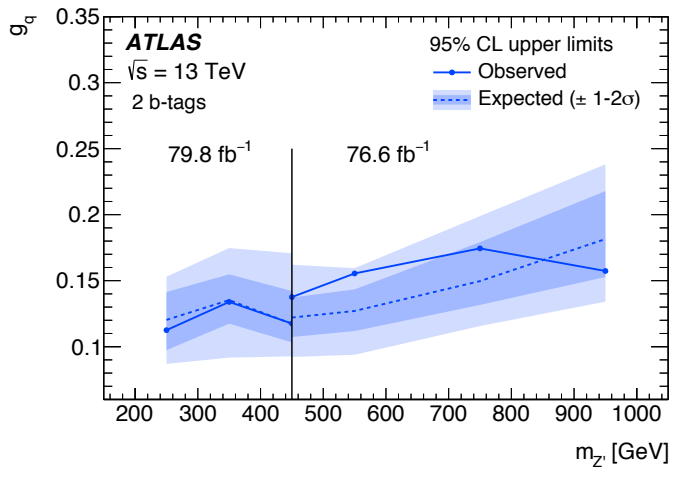

(b)

Figure 2: Upper limit at 95\% CL on the coupling of an axial-vector $Z^{\prime}$ boson to quarks as a function of its mass for (a) a flavour-inclusive selection and (b) a selection that requires two $b$-tags [3].

The multijet background was suppressed by requiring $\left|y^{*}\right|<0.75$, and was again estimated with the sliding-window method. In addition to analysing $m_{j j}$ regardless of the flavour of the two jets, a selection with two $b$-tags was studied, using the two highest- $p_{\mathrm{T}} b$-tagged jets. No significant excess above the SM background was found and 95\% CL upper limits were set on the coupling of an axial-vector $Z^{\prime}$ boson to quarks as a function of its mass (Figure 2). This $Z^{\prime}$ boson appears in simplified dark matter (DM) models as a mediator particle [4] and was here searched for in the decay to SM particles instead of DM particles. At lower masses, the best limits were obtained with the single-photon trigger, at higher masses, the best limits were obtained with the combined trigger ${ }^{1}$. Although the coupling of the $Z^{\prime}$ to SM quarks is assumed to be the same for all quark flavours, the limits in the case of the selection with $b$-tags are slightly better than in the case of the flavour-inclusive selection, because requiring two $b$-tags efficiently suppresses the multijet

\footnotetext{
${ }^{1}$ The combined trigger was not present during 2015 data taking. The dataset taken with this trigger hence corresponds to $76.6 \mathrm{fb}^{-1}$ of data only.
} 
background. Also in this search, limits were additionally set on a generic signal with a Gaussian shape in $m_{j j}$.

When searching for even lower-mass resonances, the minimum $p_{\mathrm{T}}$ of the ISR trigger objects results in an average Lorentz boost of the dijet resonance that is large enough for the hadronisation products of the two partons to merge. Hence, instead of searching for a resonance of two jets with a small jet-radius parameter, a resonance in the invariant mass of one jet with a large radius parameter (large- $R$ jet), expected to contain both partons, was searched for using $80.5 \mathrm{fb}^{-1}$ of data [5]. In this search, the trigger object was an ISR jet, expected for example from gluon radiation from the incoming partons. Two $b$-tags were required to be associated with the large- $R$ jet. The shape of the dominant multijet background was estimated in a control region (CR) without $b$-tags. Background contributions from $V+$ jets, where $V$ is either a $W$ or $Z$ boson, $t \bar{t}$, and SM Higgs-boson production were estimated using Monte Carlo (MC) simulations.

No significant excess above the SM background was found and 95\% CL upper limits were again set on the coupling of an axial-vector $Z^{\prime}$ boson to quarks as a function of its mass (Figure 1(b)). These results extend those from the photon-triggered search to lower resonance masses. The results were also used to measure simultaneously the signal strength of the SM $V+$ jets and Higgs boson processes.

\section{Top-antitop resonances}

Resonances that decay to $t \bar{t}$ were searched for in the all-hadronic $t \bar{t}$ decay mode, using $36.1 \mathrm{fb}^{-1}$ of data [6]. The all-hadronic decay mode is characterised by a large BR but also a large background from multijet production. The search was performed in the "resolved channel" in a multijet final state for low resonance masses, and in the "boosted channel" in a final state with two large- $R$ jets, each expected to contain all decay products of a high- $p_{\mathrm{T}}$ hadronically-decaying top quark, for high resonance masses. The multijet background was efficiently suppressed by $b$-tagging requirements and by requirements on the quality of top-quark reconstruction. For this reconstruction, in the resolved analysis, the "buckets of tops" algorithm [7] was used to sort the jets in an event into different "buckets", comparing the invariant mass of each bucket to the masses of the top quark and of the $W$ boson. In the boosted analysis, top-tagging based on the mass of the large- $R$ jet and on the compatibility of its substructure with a three-prong decay were used to distinguish jets from top quarks and from background processes. The quality of the top-quark reconstruction and the presence of $b$-tags were used to categorise events into signal regions (SR) and CRs, used for the estimate of the multijet background from data.

No significant excess above the Standard Model (SM) background was found and 95\% CL upper limits were set on the cross section times BR of a topcolor-assisted technicolor $Z^{\prime}$ boson [8,9] as a function of its mass (Figure 3(a)). Although the all-hadronic final state intrinsically comes with a much larger total background, this analysis is competitive to a search for $t \bar{t}$ resonances in the lepton+jets $t \bar{t}$ decay mode, published earlier [10] (Figure 3(b)). The excellent sensitivity of the all-hadronic search stems from the efficient suppression of the multijet background using topreconstruction techniques and $b$-tagging. Limits were also set on vector- and axial-vector mediators from simplified DM models, and on Kaluza-Klein gluons. 


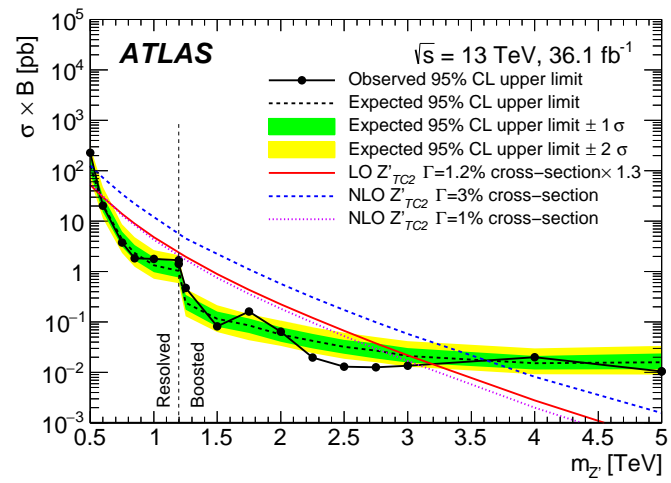

(a)

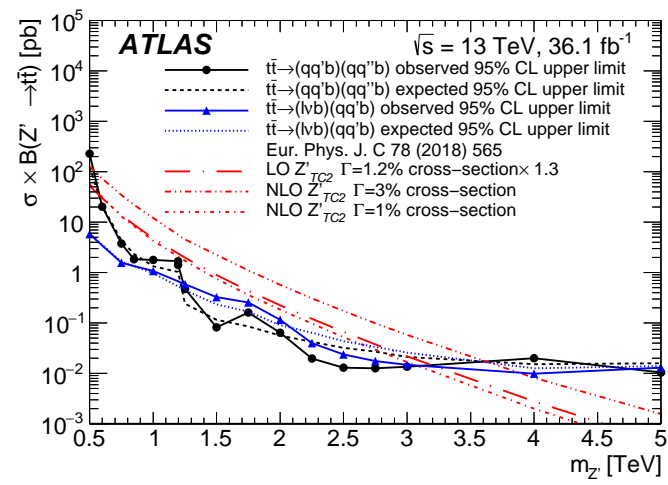

(b)

Figure 3: Upper limit at 95\% CL on the cross section times branching ratio of a topcolor-assisted technicolor $Z^{\prime}$ boson, $Z_{\mathrm{TC} 2}^{\prime}$, as a function of its mass (a) for the all-hadronic analysis [6] and (b) comparing [6] the all-hadronic analysis and the analysis in the lepton+jets channel [10].

\section{Vector-like quarks}

VLQs are often assumed to couple primarily to the third SM quark generation, in which case they are referred to as "top partners". As they are colour-charged particles, they could be produced in pair production via the strong interaction at the LHC. If only decays to SM particles are allowed, they are predicted to decay to a massive boson and a third-generation quark. For example, vector-like $T(B)$ quarks would have allowed BRs to $H t(H b), Z t(Z b)$ and $W b(W t)$, resulting in a multitude of final states that can be used to search for VLQs. Six searches for vector-like $T$ quarks and four searches for vector-like $B$ quarks using $36.1 \mathrm{fb}^{-1}$ of data were combined to enhance the overall sensitivity to VLQs [11]. For any combination of BRs to SM particles, vector-like $T(B)$ quarks with masses lower than $1.31 \mathrm{TeV}(1.03 \mathrm{TeV})$ were excluded at 95\% CL.

With the lower mass limits for VLQs now larger than $1 \mathrm{TeV}$, the search for VLQ single production gains in importance, because the single-production cross section can be significantly larger than the pair-production cross section for large VLQ masses [12]. Single production of vector-like $T$ quarks was searched for using $36.1 \mathrm{fb}^{-1}$ of data in the $T \rightarrow W b$ decay mode [13] and in the $T \rightarrow Z t$ decay mode with the subsequent decays $Z \rightarrow v \bar{v}$ and $t \rightarrow W^{+} b \rightarrow q \bar{q}^{\prime} b$ [14].

In the $T \rightarrow W b$ analysis, the SR was defined using an electron or muon, missing transverse momentum $\left(E_{\mathrm{T}}^{\text {miss }}\right)$, a $b$-tagged jet, and a forward jet from the $t$-channel production. In the $T \rightarrow Z t$ analysis, the SR was defined using a top-tagged large- $R$ jet recoiling against a large amount of $E_{\mathrm{T}}^{\mathrm{miss}}$, and again a forward jet from the $t$-channel production. In both analyses, the dominant background contributions were estimated using MC simulations and validated using data CRs. In the $T \rightarrow W b$ analysis, the $W+$ jets and single-top backgrounds were found to interfere with the signal. The dependence of the interference effects on the electroweak coupling to the $W$ boson was taken into account in the interpretation of the results. In both analyses, no significant excess was found, and 95\% CL upper limits were set on the electroweak coupling to the $W$ boson as a function of the mass of the vector-like $T$ quark (Figure 4). 


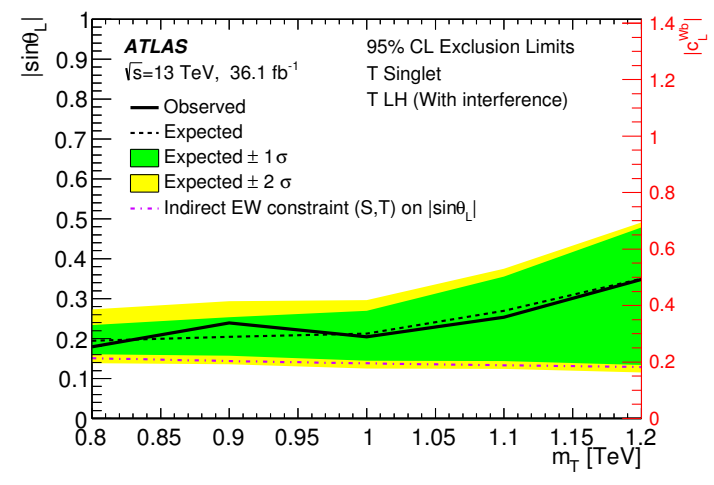

(a)

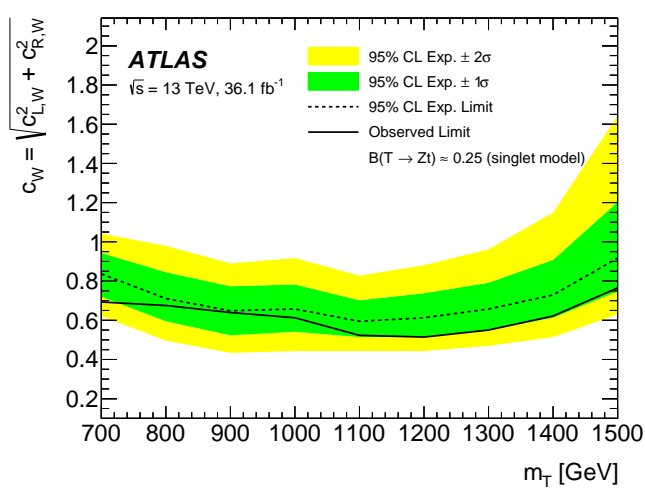

(b)

Figure 4: Upper limit at 95\% CL on the electroweak coupling of the vector-like $T$ quark to the $W$ boson as a function of its mass in the searches using (a) the $T \rightarrow W b$ decay mode [13] and (b) the $T \rightarrow Z t$ decay mode [14].

\section{Summary}

The results from a selection of searches for dijet resonances, for resonances that decay to a top quark and an antitop quark, and for vector-like quarks were presented. The data were taken with the ATLAS detector in $13 \mathrm{TeV}$ proton-proton collisions at the LHC. No significant excess above the Standard Model background was found and limits were set on the parameters of benchmark models for the respective final states.

\section{References}

[1] ATLAS Collaboration, JINST 3 (2008) S08003

[2] ATLAS Collaboration, ATLAS-CONF-2019-007, cdsweb.cern.ch/record/2668385

[3] ATLAS Collaboration, 1901.10917 [hep-ex]

[4] D. Abercrombie et al., 1507.00966 [hep-ex]

[5] ATLAS Collaboration, ATLAS-CONF-2018-052, cdsweb.cern.ch/record/2649081

[6] ATLAS Collaboration, Phys. Rev. D 99 (2019) 0920041902.10077 [hep-ex]

[7] M. R. Buckley et al., JHEP 08 (2013) 086, 1302 . 6238 [hep-ph ]

[8] C. T. Hill and S. J. Parke, Phys. Rev. D 49 (1994) 4454 [hep-ph / 9312324$]$

[9] C. T. Hill, Phys. Lett. B 345 (1995) 483 [hep-ph / 9411426 ]

[10] ATLAS Collaboration, Eur. Phys. J. C 78 (2018) 565 [1804.10823 [hep-ex] ]

[11] ATLAS Collaboration, Phys. Rev. Lett. 121 (2018) 211801 [1808.02343 [hep-ex] ]

[12] J.-A. Aguilar-Saavedra et al., Phys. Rev. D 88 (2013) 094010 [1306.0572 [hep-ph] ]

[13] ATLAS Collaboration, 1812.07343 [hep-ex]

[14] ATLAS Collaboration, JHEP 05 (2019) 411812.09743 [hep-ex ] 